\title{
Responsible Gambling Through a Motivational Telephone Intervention to High-risk Gamblers - an Evaluation of User Satisfaction and Subjective Intervention Effects
}

Anders Håkansson ( $\nabla$ anders_c.hakansson@med.lu.se )

Lund University

Katja Franklin

AB Svenska Spel

Maria Dahlström

AB Svenska Spel

Axel Lyckberg

AB Svenska Spel

\section{Research Article}

Keywords: gambling disorder, motivational interviewing, responsible gambling

Posted Date: February 24th, 2022

DOI: https://doi.org/10.21203/rs.3.rs-1349386/v1

License: (c) (i) This work is licensed under a Creative Commons Attribution 4.0 International License.

Read Full License 


\section{Abstract}

Background: Problem gambling causes major social and psychiatric consequences, and there is need to develop feasible harm-reducing or therapeutic interventions. It has been suggested that gambling operators with responsible gambling intentions can intervene in order to identify and help reduce the harm in problem gamblers. However, user satisfaction, and the risk of negative reactions and gamblers' transfer to other operators, rarely have been explored scientifically.

Methods: This is a retrospective survey study of gamblers reached by a motivational, telephone intervention by the Swedish state-owned gambling operator (Svenska Spel), addressing gamblers with indicators of high-risk gambling practices $(n=197)$. Surveys were answered approximately 10 days after the intervention.

Results: Thirty-seven percent perceived their gambling to have decreased due to the intervention, whereas seven percent perceived their gambling instead had increased. A large majority (80 percent) reported a subjective favorable experience from the intervention. Forty percent reported gambling on other operators than Svenska Spel after the intervention, but only seven percent gambled on a new operator following the intervention.

Conclusions: Survey data in gamblers reached with a motivational telephone intervention present mainly promising findings, and should be replicated in future studies in larger samples. The main findings display an overwhelmingly favorable subjective experience, and a limited risk of gamblers migrating to other operators.

\section{Background}

Problem gambling presents a health hazard world-wide, with severe social and mental health-related consequences, but is also a condition which can be prevented or treated [1, 2]. A number of low-threshold harm-reducing or therapeutic interventions have been suggested [3], including those delivered directly by a gambling operators with responsible gambling policies [4]. These may, for example, involve mandatory play breaks, direct automated messaging with personalized feedback on gambling patterns [5], or general consumer protection information providing links to different harm-reducing measures, such as voluntary self-exclusion or limit setting [3]. However, a more direct contact from a gambling operator, such as in a personalized telephone call aiming to reach gamblers at risk with responsible gambling measures, is far less common [4].

As part of its responsible gambling strategy, the state-owned gambling operator AB Svenska Spel, in Sweden, has introduced systematically a motivational telephone intervention to gamblers who either present high levels of gambling losses, or who screen positive for gambling problems defined as a 'red' alert level on a self-assessment tool (GamTest, [6]) delivered through the gambling platform. The present intervention project has been described previously [7]. 
Follow-up data describing post-intervention gambling, limit setting and self-exclusion, comparing individuals reached with the intervention and those who were not reached (control group), has been analyzed and are published elsewhere. Likewise, in a similar project in Norway, the state-owned gambling operator carried out motivational contact attempts addressing subjects with intense gambling practices, in that case defined as the proportion of the operator's clients with the highest net losses. Here, a personal telephone intervention was more efficacious in reducing high-risk gambling practices, compared to a postal letter contact or a control group [8, 9]. Both analyses, however, are limited by the fact that only the own operator's gambling data can be measured, such that gambling on either gambling operators cannot be assessed. Thus, even in subjects with reduced or discontinued gambling on the gambling operator which intervenes, it is still possible that further gambling may occur at other operators. Likewise, studies hitherto have not been able to assess, retrospectively, how included individuals perceive and experience the intervention, and which subjective effects the intervention may have on gambling behaviors.

For example, the gambling market in Sweden involves a larger number of licensed gambling operators, many of which offer online games involving either online casino or online sports betting, i.e. gambling types which are easily accessible to individuals who may increase their gambling practices on other operators, after receiving a responsible gambling intervention in one operator. It shall be borne in mind, for example, that recently, a COVID-related governmental preventive intervention in Sweden addressed people with high gambling losses on online casino in one operator, and where it was shown that many people with high-risk gambling practices instead turned to other online operators thereafter [10]. Also, although it may not be common [11], it is also possible that gamblers reached with a motivational intervention may react with anger, potentially with a deteriorating effect on their gambling practices. In particular, while responsible gambling tools have not been shown to cause irritation and to scare off gamblers in the segment of non-problem and moderate-risk gamblers, such negative reactions were shown to be significantly more common in problem gamblers [11]. Therefore, in an intervention aiming to help high-risk gamblers reduce or discontinue their gambling, it is of utter importance to assess individuals' subjective experience of such intervention.

For these reasons, the present study was carried out in gamblers who had been reached by AB Svenska Spel in a motivational telephone intervention, aiming to assess their 1) subjective user satisfaction with the intervention, 2) their subjective opinion about how the intervention had changed their gambling behavior following the intervention, 3) gambling on the own operator and on other gambling operators following the intervention, including the potential risk of migrating to a new gambling operator after the present responsible gambling intervention, and 4) whether user satisfaction with the intervention was associated with changes in gambling and potential gambling on other operators post-intervention.

\section{Methods}

The study involved individuals who had undergone the motivational intervention [7], either based on a 'red' self-test (GamTest, [6]), or based on high levels of detected gambling losses (although cut-off levels have varied during the study period, as previously reported [7]. In brief, the motivational calls contained 
the following; information about the intervention and permission to proceed, questions and information about the client's losses and her/his reactions to this information, reflective listening and continuation of the call depending on the client's motivational, change talk, and in case of the client's permission, further information and discussion about responsible gambling tools such as limit settings or self-exclusion, as well as a discussion around the client's plans for future gambling behaviors. The telephone conversations had a motivational, non-judgmental, and reflective listening approach. Study subjects were contacted 10 days after the call. The study was carried out from March 23, 2021, through October 1, 2021. A total of 900 subjects were invited, and 204 complete responses (and 39 partial responses) were collected.

Surveys were opened only in case an individual provided informed consent in the electronic format of the survey. For a completed study response, an individuals received a compensation consisting of an electronic gift card with a value of 100 SEK (a gift card possible to use in a number of stores, although not for the purchase of alcohol, tobacco, or gambling).

A number of responses derived from the same individuals, who had responded both with a complete or a partial survey response. Here, only the first survey was kept, and thereafter, the final sample size was 197 individuals with a complete study answer.

The study was approved by the Swedish Ethics Authority (application file number 2020-03281). It was presented in a protocol paper [7], and pre-registered at clinicaltrials.gov (identifier NCT04646421).

\section{Measures}

Questions referred to the telephone intervention carried out, and the items describing the subject's subjective experience of the intervention were based on the following questions, which were rated on a 5point scale ranging from 'fully agree' to 'fully disagree' (Table 1): the telephone call 1) makes me feel the operator cares about me, 2) makes me angry or annoyed, 3) made me decrease my gambling after the call, 4) made me increase my gambling after the call, 5) gave me primarily a favorable experience, and 6) gave me primarily an unfavorable experience. 
Table 1

Subjective patient satisfaction with the motivational telephone intervention ( $N=197)$.

\begin{tabular}{|lllllll|}
\hline & $\begin{array}{l}\text { Call makes } \\
\text { me feel the } \\
\text { operator } \\
\text { cares about } \\
\text { me, } \mathbf{n}(\%)\end{array}$ & $\begin{array}{l}\text { Call } \\
\text { makes } \\
\text { me } \\
\text { annoyed } \\
\text { or angry, } \\
\mathbf{n}(\%)\end{array}$ & $\begin{array}{l}\text { Call } \\
\text { decreased } \\
\text { my } \\
\text { gambling } \\
\text { after the } \\
\text { call, } \mathbf{n}(\%)\end{array}$ & $\begin{array}{l}\text { Call } \\
\text { increased } \\
\text { my } \\
\text { gambling } \\
\text { after the } \\
\text { call, } \mathbf{n}(\%)\end{array}$ & $\begin{array}{l}\text { Call gave } \\
\text { me primarily } \\
\text { a favorable } \\
\text { experience, } \\
\mathbf{n}(\%)\end{array}$ & $\begin{array}{l}\text { Call gave me } \\
\text { primarily an } \\
\text { unfavorable } \\
\text { experience, } \mathbf{n} \\
(\%)\end{array}$ \\
\hline $\begin{array}{l}\text { Fully } \\
\text { agree }\end{array}$ & $132(67)$ & $8(4)$ & $20(10)$ & $7(4)$ & $103(52)$ & $6(3)$ \\
\hline $\begin{array}{l}\text { Partly } \\
\text { agree }\end{array}$ & $42(21)$ & $18(9)$ & $53(27)$ & $6(3)$ & $54(27)$ & $9(5)$ \\
\hline $\begin{array}{l}\text { Neither } \\
\text { Partly }\end{array}$ & $13(7)$ & $26(13)$ & $69(35)$ & $45(23)$ & $29(15)$ & $33(17)$ \\
\hline disagree & $5(3)$ & $26(13)$ & $15(8)$ & $24(12)$ & $8(4)$ & $34(17)$ \\
\hline $\begin{array}{l}\text { Fully } \\
\text { disagree }\end{array}$ & $5(3)$ & $119(60)$ & $40(20)$ & $115(58)$ & $3(2)$ & $115(58)$ \\
\hline
\end{tabular}

The subsequent questions asked whether the subject, since the telephone intervention, had self-excluded on the nationwide Swedish self-exclusion service 'Spelpaus' [12], had gambled on a different gambling operator than Svenska Spel, and if so, whether the subject had gambled on an operator on which she/he had never gambled before.

Thereafter, the subject was asked about which gambling types she/he had gambled at any time during the past year prior to the intervention, and at any time after the telephone intervention, respectively. Gambling types were collapsed into the categories of sports betting (on any operator), horse race betting (on any operator), online poker gambling (on any operator), and online casino/bingo gambling (including online bingo and online casino on any operator, Table 1). The following items asked about whether the subject had gambling-related debts, whether she/he had any involvement with the Swedish enforcement agency due to debts, and questions about current, occupation (work, studying, retired, sick-leave, or unemployed), gender (female/male), and age group ( $<25,25-29,40-59$, or $>59$ years).

\section{Statistical methods}

A comparison was made between subjects who reported having self-excluded from gambling after the intervention vs those who had not, between those reporting decreased gambling after the intervention vs all others, those reporting gambling on other operators after the intervention vs others, and between those reporting gambling on a new operator after the intervention vs all others. These comparisons were made using chi-square tests (Fisher's exact whenever the number in any of the groups was five or lower). Regarding self-reported decrease in gambling after the intervention, and regarding any gambling on other operators after the intervention, these outcome variables were analyzed in logistic regression analyses, including age group, gender, and each of the gambling types included (online casino/bingo gambling, 
sports betting, horse race betting, and online poker). Due to the limited statistical power and the low number of individuals endorsing some outcomes, no logistic regression analysis was carried out for gambling on a new operator after the intervention, for the reporting of having self-excluded after the intervention, or for the perception of having gambled more after the intervention.

\section{Results}

A majority of included individuals were male (93 percent, $n=183$ ), and working (75 percent, $n=147$ ) or studying (10 percent, $n=20)$, whereas seven percent $(n=13)$ were retired, five percent $(n=10)$ were unemployed, and four percent $(n=7)$ were on sick-leave. A total of 16 percent $(n=32)$ had gamblingrelated debts, and 17 percent $(n=34)$ had debts involving the national enforcement authority. The largest age groups were 40-59 years (42 percent, $n=83$ ), and 25-39 years ( 26 percent, $n=51$ ), respectively, whereas 19 percent $(n=38)$ were younger than 25 years, and 13 percent $(n=25)$ were 60 years or older.

A large majority of respondents (88 percent) perceived the telephone intervention as a sign of the operator caring about the gambler, and a minority, 13 percent of respondents, endorsed being annoyed or angry with the intervention. Regarding gambling after the intervention, 37 percent reported that they perceived their gambling to have decreased because the intervention, whereas a minority (seven percent) reported that their gambling had increased because of the intervention. A large majority (80 percent) reported a mainly favorable experience of the telephone intervention, and few (eight percent) a mainly unfavorable experience (Table 1).

Gambling data reported for the past-year period prior to the intervention, and for the period after the intervention, respectively, are reported in Table 2. 
Table 2

Self-reported gambling patterns for the past year prior to the intervention, and for the time period during the intervention and the survey (approximately 10 days), respectively $(\mathrm{N}=197)$.

\begin{tabular}{|lll|}
\hline & $\begin{array}{l}\text { Past-year gambling prior to the } \\
\text { intervention, } \mathbf{n}(\%)\end{array}$ & $\begin{array}{l}\text { Gambling after the } \\
\text { intervention, } \mathbf{n}(\%)\end{array}$ \\
\hline $\begin{array}{l}\text { Sports betting on Svenska } \\
\text { Spel }\end{array}$ & $176(89)$ & $156(79)$ \\
\hline $\begin{array}{l}\text { Sports betting on other } \\
\text { operators }\end{array}$ & $102(52)$ & $71(36)$ \\
\hline Horse race betting & $97(49)$ & $63(32)$ \\
\hline Svenska Spel online casino & $93(47)$ & $70(36)$ \\
\hline Other operators' online casino & $81(41)$ & $52(26)$ \\
\hline Land-based casino & $19(10)$ & $4(2)$ \\
\hline Online poker & $45(23)$ & $30(15)$ \\
\hline Online bingo & $25(13)$ & $9(5)$ \\
\hline Land-based bingo & $4(2)$ & $5(3)$ \\
\hline Restaurant casino & $16(8)$ & $8(4)$ \\
\hline $\begin{array}{l}\text { Land-based electronic } \\
\text { gambling machines }\end{array}$ & $44(22)$ & $21(11)$ \\
\hline
\end{tabular}

Seven percent $(n=13)$ reported that they had self-excluded from gambling after the intervention. Those who had self-excluded were significantly younger $(p=0.03$, chi-square linear-by-linear, with 31 percent in the youngest age group vs 18 percent among the remaining respondents), whereas they did not differ significantly with respect to the number of sports bettors ( 85 vs 91 percent, $p=0.36$, Fisher's exact test), online casino/bingo gamblers (69 vs 52 percent, $p=0.26$ Fisher's exact test), horse race bettors (54 vs 49 percent, $p=0.78$, Fisher's exact test), online poker gamblers (23 vs 23 percent, $p=1.00$, Fisher's exact test), or men (85 vs 93 percent, $p=1.00$, Fisher's exact test).

Forty percent $(n=79)$ reported that they had gambled on a different gambling operator (than Svenska Spel) after the intervention. Among them, 16 percent $(n=13$, seven percent of all respondents) reported that they had gambled on an operator on which they had never gambled before. Those who had gambled on a new operator after the intervention were significantly younger $(p<0.001$, chi-square linear-by-linear, with 54 percent in the youngest age group vs 17 percent among the remaining respondents), whereas they did not differ significantly with respect to the number of sports bettors (77 vs 91 percent, $p=0.12$, Fisher's exact test), online casino/bingo gamblers (77 vs 52 percent, $p=0.09$ Fisher's exact test), horse race bettors (31 vs 51 percent, $p=0.25$, Fisher's exact test), online poker gamblers (15 vs 23 percent, $p=$ 0.74 ), or men (92 vs 93 percent, $p=1.00$, Fisher's exact test). 
Gamblers who reported having mainly a favorable experience of the telephone intervention were significantly more likely to report that their gambling decreased after the intervention (43 vs 15 percent, $p$ $<0.01$ ), and less likely to report gambling on other operators after the intervention (36 vs 55 percent, $p=$ $0.03)$. They did not differ significantly from other subjects with respect to self-exclusion after the intervention ( 8 vs 3 percent, $p=0.24$ ) or the reporting of having gambled on a new gambling operator after the intervention ( 5 vs 13 percent, $p=0.09$ ). Gamblers who reported being angry or annoyed by the call tended to be more likely to report gambling on an operator on which had never gambled before ( $15 \mathrm{vs}$ 5 percent, $p=0.05$ ), but were not more likely to report decreased gambling (39 vs 37 percent, $p=0.87$ ), self-exclusion (8 vs 6 percent, $p=0.81$ ), or any gambling on a different operator than Svenska Spel (39 vs 40 percent, $p=0.85)$.

In logistic regression, a self-reported decrease in gambling after the intervention was not significantly associated with the gender (OR 0.49 for male gender [0.12-2.06], $p=0.33$ ), age group (OR 0.98 [0.691.38], $p=0.89$ ), or with any of the specific gambling types assessed (OR 1.76 for online casino/bingo gambling [0.94-3.31], $p=0.08$, OR 2.33 for sports betting [0.59-9.17], $p=0.23$, OR 1.07 for horse race betting [0.58-1.97], $p=0.83$, and OR 0.73 for online poker [0.34-1.55], $p=0.41$ ). Also, in logistic regression, self-reported gambling on other operators after the intervention was not significantly associated with gender (OR 1.01 for male gender [0.25-4.12], $p=0.99$ ), age group (OR 0.81 [0.58-1.15], $p=0.24$ ), or with any of the specific gambling types assessed (OR 1.60 for online casino/bingo gambling [0.86-2.97], $p=0.14$, OR 0.68 for sports betting [0.20-2.35], $p=0.54$, OR 1.77 for horse race betting [0.95-3.29], $p=0.07$, and OR 1.10 for online poker [0.53-2.26], $p=0.80$ ).

\section{Discussion}

The present study is one of very few studies assessing gamblers' own subjective experience of a personalized harm-reducing intervention delivered by a gambling operator. It is one part of a pre-defined follow-up evaluation of a responsible gambling intervention carried out by the state-owned gambling operator of Sweden [7]. Despite limitations related to the limited sample size and a low response rate, this study lends support to a personalized, motivational telephone intervention addressing clients with highrisk gambling practices. In a sample with extensive past-year gambling habits, a large majority reported a favorable impression of the intervention, and a substantial percentage reported, subjectively, that the intervention decreased their gambling practices.

Although the present survey was carried out less than two weeks after the telephone intervention, a nonnegligeable number of clients had self-excluded from gambling. Although the conclusion to be drawn from this are limited, due to the absolute lower numbers, it is of importance to study further whether younger individuals are more likely to self-exclude after this type of motivational intervention, as in the present study. This being said, however, the main finding of the study is likely the fact that four out of five respondents perceived the intervention as mainly favorable, and that only eight percent perceived it as negative, and only seven percent reported that it increased their gambling. Also, it is of importance to note that individuals who had a favorable impression of the intervention were both more likely to decreases 
their gambling and less likely to gamble on other operators after the intervention, which further strengthens the impression of the intervention being feasible in the present population.

Also, only a limited minority of respondents (seven percent of the whole study sample) reported that they gambled on new operators after the telephone intervention. Thus, this data speaks against the fear that this type of intervention - a personalized, harm reduction-oriented intervention to clients with possible gambling problems - would annoy people and to have a counter-productive effect of increased gambling practices or to push gambler towards other operators with less defined responsible gambling practices. Although this is a promising finding, it has to be replicated in larger studies, preferably with higher response rate, in order to rule out the risk that people with a more favorable approach towards the intervention may be more likely to respond to the survey. Also, and despite the fact that the low absolute numbers of respondents were low, clients who reported a negative reaction to the call were significantly more likely to report gambling on an operator on which they had never gambled before. This may corroborate with the findings of Ivanova and co-workers [11], where a subgroup with gambling problems had more unfavorable attitudes towards responsible gambling practices, and may be a sign of the challenge of intervening in the minority of respondents who gave a more negative description of the intervention. On the contrary, however, clients who reporting being angry or annoyed by the call still were not more likely to report increased gambling. Thus, while the presence of other gambling operators on the market is a reality [10], with a certain risk of a subgroup changing to other operators after a responsible gambling intervention, they may not necessarily worsen their gambling behavior and they may still retain motivational components from the conversation. Further longitudinal studies are needed in order to fully outline changes in gambling behaviors in different subgroups reached by thee present type of intervention.

The group of clients assessed in the present study had a high gambling involvement and likely high rates of gambling problems and gambling-related harm. Around one out of six participants reported gamblingrelated debts and debts involving the national enforcement agency. Data for comparison are few, but in a recent online survey of online gamblers in Sweden, nine percent reported gambling-related borrowing, but this was highly correlated with gambling problems; nine out of ten of the subjects reporting such borrowing were moderate-risk or problem gamblers. Expressed in the opposite way; among problem gamblers in the study, 50 percent had ever borrowed money for gambling. In the same study, eight percent of online gamblers were involved with the enforcement agency, but this figure was as high as 16 percent in sports and casino gamblers, and 13 percent in those reporting online casino gambling but no sports betting [13]. Thus, gambling for borrowed money, and thereby the risk of indebtedness, appears to be common in online gamblers with the most intense gambling practices. This may explain the relatively high rates of debts and enforcement involvement in the present study, where gamblers were contacted due to signs of at-risk gambling practices and where many gamblers had a gambling pattern predominantly occurring online.

Altogether, the present study adds to and resembles the conclusions by Ivanova and co-workers [11], who reported that negative reactions to a responsible gambling intervention were possible but rare. Also, the 
study adds to the promising findings seen in studies conducted with the Norwegian state-owned gambling operator, and where a telephone intervention was more effective in reducing gambling problems than a control condition or a postal intervention $[4,8,9]$. Thus, the study adds to the rationale behind introducing active harm-reducing intervention programs in other gambling operators. In particular, given the low number of respondents who stated a clearly negative experience from the intervention, it appears to be rational for gambling operators to pursue identification of gamblers with hazardous gambling practices, and to carry out a personalized intervention with the aim to help reduce their gambling practices, or to facilitate self-exclusion, initiate a motivational process possibly leading to treatment seeking. Likewise, the study may lend support to policy makers implying regulations on gambling operators on measures to be taken whenever a problematic gambling behavior is detected. For example, the Swedish government has stated, in a gambling act effective since 2019, that gambling operators are to take responsible gambling measures when gambling problems are suspected [14]. While such initiative need to be implemented and adapted with respect to feasibility and effectiveness, the present study may provide some preliminary evidence that active, personalized interventions as part of that kind of responsible gambling policy are at least unlikely to have a negative impact on gamblers, and more likely may provide an important step in the motivational process of at-risk gamblers.

The present study has limitations. First, based on the defined criteria of high-risk gambling of the present project, a very large percentage of gamblers were men, and for that reason, results or predictors of outcome could not be analyzed for women and men separately. Also, a minority of clients addressed with the intervention could be included in the present study, based on a low response rate. For this reason, results cannot be generalizable to any gambler reached by this intervention, and also cannot be generalizable to individuals with high-risk gambling practices in general. Thus, it cannot be excluded, for example, that subjects with more favorable attitudes towards the intervention were more likely to take the survey. Also, from a statistical standpoint, the final sample size was likely insufficient for potential subgroup differences to be seen. In regression analyses of a self-reported decrease in gambling, and analyses of gambling on other gambling operators after the intervention, did not display significant predictors for these outcome measures, and it cannot be excluded that such potentially significant interventions would have been hidden by low statistical power. For example, online casino/bingo gambling displayed some non-significant tendency to increase the likelihood of a gambling-decreasing effect after the intervention, and such a potential association would have required a larger study sample to be fully ruled out or confirmed. Likewise, in the non-adjusted analyses of the small groups with rare study outcomes, such associations could not be controlled for other variables in a larger regression analysis. Both clients who self-excluded from gambling, and clients who reported initiating gambling on other operators, were significantly younger. This would possibly indicate a more volatile gambling pattern in the youngest age group, i.e. both this a higher likelihood of a more favorable or a clearly unfavorable self-reported outcome. Thus, a responsible gambling intervention, in the subgroup of young individuals, may both have a higher and lower likelihood of reaching a favorable result, compared to older individuals. For example, it may be hypothesized that migration between gambling types or between operators may be larger and therefore could explain this pattern in the young, but the testing of such a hypothesis goes 
beyond the scope of the present study. Future studies should address larger study samples, in order to better highlight whether these age differences remain after controlling for other variables of relevance.

\section{Declarations}

\section{Funding}

The present work was financed by the research funding of the first author, which comes from AB Svenska Spel and from the regional health care system, and from by resources from AB Svenska Spel directly.

\section{Data availability statement}

Based on the ethics approval behind the study, data cannot be shared with undue reservation. Request for data can be sent to the first author, and data in non-identified format can be made available after review by the ethics authority and by the gambling operator AB Svenska Spel which owns the data.

\section{Conflicts of interest}

Anders Håkansson is employed by Lund University, as a full professor in addiction medicine with specialization in problem gambling. His position is financially supported by AB Svenska Spel, which is the government-owned gambling operator of Sweden. All other authors of the paper are employed by $A B$ Svenska Spel.

\section{Author contributions}

All authors contributed to the overall research idea and planned the study together. Data collection was carried out by all authors together. Statistical analyses were carried out by Håkansson, who also wrote the draft of the paper. All authors contributed to the manuscript and approved the final version for submission.

\section{References}

1. Potenza MN, Balodis IM, Derevensky J, Grant JE, Petry NM, Verdejo-Garcia A, Yip SW. Gambling disorder. Nat Rev Dis Prim. 2019;5:51.

2. Di Nicola M, De Creszenzo F, D’Alò GL, Remondi C, Panaccione I, Moccia L, Molinaro M, Dattoli L, Lauriola A, Martinelli S, Giuseppin G, Maisto F, Crosta ML, Di Pietro S, Amato L, Janiri L.

Pharmacological and psychosocial treatment of adults with gambling disorder: a meta-review. J Addict Med. 2020;14:e15-23.

3. Gainsbury SM, Angus DJ, Procter L, Blaszczynski A. Use of consumer protection tools on internet gambling sites: customer perceptions, motivators, and barriers to use. J Gambl Stud. 2020;36:25976. 
4. Jonsson J, Hodgins DC, Munck I, Carlbring P. Reaching out to big losers: a randomized controlled trial of brief motivational contact providing gambling expenditure feedback. Psychol Addict Behav. 2019;33:179-89.

5. Hopfgartner N, Auer M, Santos T, Helic D, Griffiths MD. The effect of mandatory play breaks on subsequent gambling behavior among Nowegian online sports betting, slots and bingo players: a large-scale real world study. J Gambl Stud. 2021 [online ahead of print].

6. Forsström D, Lindner P, Jansson-Fröjmark M, Hesser H, Carlbring P. GamTest: psychometric evaluation in a low-gambling general population. J Gambl Iss. 2020;44:77-102.

7. Håkansson A, Dahlström M, Franklin K, Lyckberg A. Responsible gambling telephone intervention to high-risk gamblers by a state-owned gambling operator in Sweden: study protocol for a study on effectiveness, user satisfaction, and acceptability. Int J Environ Res Publ Health 2020;17

8. Jonsson J, Hodgins DC, Munck I, Carlbing P. Reaching out to big losers leads to sustained reductions in gambling over 1 year: a randomized controlled trial of brief motivational contact. Addiction 2020;115:1522-31.

9. Jonsson J, Hodgins DC, Munck I, Carlbring P. Reaching out to big losers: how different types of gamblers are affected by a brief motivational contact initiated by the gambling provider. J Gambl Stud. 2021;37:387-401.

10. Håkansson A, Sundvall A, Lyckberg A. Effects of a national preventive intervention against potential COVID-19 gambling problems: a self-report survey in online gamblers. JMIR Form Res. 2021 [e-pub ahead of print].

11. Ivanova E, Rafi J, Lindner P, Carlbring, P. Experiences of responsible gambling tools among nonproblem gamblers: a survey of active customers of an online gambling platform. Addict Behav Rep. 2019;9:100161.

12. Håkansson $A$, Henzel $V$. Who chooses to enroll in a new national gambling self-exclusion system? A general population survey in Sweden. Harm Reduct J. 2020;17:82.

13. Håkansson A, Widinghoff C. Over-indebtedness and problem gambling in a general population sample of online gamblers. Front Psychiatry (2020) 11:7.

14. Government of Sweden. Swedish Gambling Act. 2018:1138. Government of Sweden, 2018. https://www.riksdagen.se/sv/dokument-lagar/dokument/svensk-forfattningssamling/spellag20181138_sfs-2018-1138 (in Swedish, accessed January 28, 2022). 